\title{
Problématiques de la couverture adéquate en penta 3 chez les enfants de $0-11$ mois dans le Centre de santé Communautaire et Universitaire de Konobougou, Mali
}

\section{Problematies of adequate coverage in penta 3, in children fron 0 to 11 mouths in Konobougou community and university health center, Mali}

\author{
Dembélé $\mathrm{K}^{1}$, Coulibaly $\mathrm{MB}^{1^{*}}$, Diarra $\mathrm{B}^{2}$, Niaré $\mathrm{B}^{3}$, Coulibaly $\mathrm{KB}^{4}$, Sidibé $\mathrm{DM}^{5}$, Kaya $\mathrm{AS}^{6}$, Sangho $\mathrm{H}^{2}$, Dembélé $\mathrm{M}^{6}$
}

\begin{abstract}
1 : Centre de Santé Communautaire et Universitaire de Konobougou (Ségou-Mali)

2 : Département d'enseignement et de recherche de Santé Publique à la FMOS de Bamako (Bamako-Mali)

3 : Direction régionale de la santé du district de Bamako (Bamako-Mali)

4 : Centre de Santé Communautaire et Universitaire de Sanoubougou II (Sikasso-Mali)

5: Centre de Santé Communautaire et Universitaire de Banconi (Bamako-Mali)

6 : Service de médecine interne du $\mathrm{CHU}$ de Point $\mathrm{G}$ de Bamako (Bamako-Mali)

*Auteur correspondant : Dr Mamadou Bayo COULIBALY, spécialiste en médecine de famille/médecine communautaire, Directeur technique du CSCom U de Konobougou, Chargé d'encadrement clinique D.E.S médecine de famille/médecine communautaire (Mali). bayo_coul@yahoo.fr ; Cel : 0022376067882

Conflit d'intérêt : Aucun
\end{abstract}

\section{Résumé}

Introduction : La mise en œuvre du Programme Elargi de Vaccination (PEV) au Mali est confrontée à plusieurs difficultés. Le but de ce travail était d'étudier les difficultés influençant la couverture adéquate en penta 3 dans l'aire du centre de santé communautaire et universitaire de Konobougou afin de proposer des alternatives pour son amélioration. Matériel et méthodes : L'étude s'est déroulée au Centre de Santé Communautaire et Universitaire de Konobougou/région de Ségou. II s'agissait d'une étude recherche action de Susman allant du 1er avril au 31 juillet 2018. Ont été inclus dans l'étude, le personnel de l'unité de vaccination, les mères/accompagnantes d'enfants à la vaccination, les relais, les leadeurs religieux et les élus locaux. Résultats : La connaissance des mères sur la tranche d'âge du PEV a évolué de $20 \%$ à $75 \%$ et celle des relais communautaires de $66,7 \%$ à $100 \%$ après l'intervention. Le plan d'action a permis d'amener de $66,7 \%$ à $100 \%$ les agents de santé formés sur l'information, l'éducation, la communication et la gestion des Manifestations Adverses Post Immunisation (MAPI). Le niveau d'implication des décideurs était de $80 \%$ au cours des deux évaluations. Conclusion : Cette étude a permis de poser des actions concrètes en impliquant les populations concernées de manière participative au cours du processus de recherche, tout en apportant des changements positifs de comportement.

Mots clés : couverture adéquate, penta 3, Konobougou, Mali

\section{Abstract}

Introduction: The implementation of the EPI in Mali faces several difficulties. The aim of this work was to study the difficulties influencing adequate penta 3 coverage in the area of the community and university health center of Konobougou in order to propose alternatives for its improvement. Material and methods: The study took place at the Community and University Health Center of Konobougou / Ségou region. This was an action research study by Susman from April 1 to July 31, 2018. The staff of the vaccination unit, mothers / caregivers of children in vaccination, relays, religious leaders and local elected officials. Results: The knowledge of mothers on the EPI age group increased from $20 \%$ to $75 \%$ and that of community workers from $66.7 \%$ to $100 \%$ after the intervention. The action plan helped bring health workers trained in information, education, communication and management of AEFI from $66.7 \%$ to $100 \%$. The level of involvement of decision makers was $80 \%$ during the two evaluations. Conclusion: This study made it possible to take concrete actions by involving the populations concerned in a participatory manner during the research process while bringing positive changes in behavior.

Keywords: adequate cover, penta 3 vaccine, Konobougou, Mali

\section{INTRODUCTION}

La survie de l'enfant est une préoccupation majeure en sante publique. Elle est constamment menacée par les maladies évitables (1). La vaccination est un acte médical préventif essentiel de santé publique dont l'efficacité est conditionnée par un taux de couverture vaccinale suffisant, pour assurer une protection individuelle et collective $(2,3)$. Elle est rentable en matière de prévention primaire (4).

Au cours du XXème siècle, la généralisation de la vaccination a fait décroître de façon spectaculaire la morbidité et la mortalité dues aux maladies infectieuses (5). Correctement administrée, elle permet de protéger l'enfant contre des maladies débilitantes sinon mortelles comme la tuberculose, la poliomyélite, la coqueluche, le tétanos, la diphtérie et la rougeole (3). Les maladies évitables sont responsables de plus de deux millions de décès par an dans le monde (1).

Le Programme Elargi de Vaccination (PEV), initié par l'organisation mondiale de la santé (OMS) en 1974, vise à lutter contre les maladies de l'enfant évitables par la vaccination (6). II permet d'éviter le décès d'au moins trois millions d'enfants chaque année et d'envisager l'éradication de la poliomyélite après celle de la variole dans le monde (1). Malgré ce résultat, on compte environ 1,5 million de décès annuels d'enfants dus à des maladies évitables par la vaccination (7). 
Le succès des programmes de vaccination dépend en grande partie de la qualité du travail des vaccinateurs et de leur attitude positive face à la vaccination. Les professionnels de la santé sont certainement le groupe le plus susceptible d'influencer la décision des parents en ce qui concerne la vaccination des enfants (8).

En Afrique subsaharienne, un enfant sur deux reçoit chaque année une série de vaccinations incomplètes (1). Les occasions de vaccination manquées constituent des obstacles majeurs à la réalisation d'une bonne couverture vaccinale dans les populations cibles du PEV. Les raisons avancées par les femmes sont diverses pour justifier leur irrégularité : manque de temps, vaccin non disponible, manque d'informations, enfants malades, etc. (9). La couverture vaccinale des enfants de moins de 5 ans, particulièrement des enfants d'un an à deux ans, est un indicateur clef de l'utilisation des services de vaccination (3).

Au Mali, selon l'EDSM V, réalisé en 2012-2013, la couverture vaccinale complète est faible et varie d'une région à l'autre. Ce taux est faible dans les régions de Ségou (38\%) (10). Les monitorages des activités du centre de santé communautaire et universitaire (CSCom U) de Konobougou réalisés du 01 ier juin au 31 décembre 2017 relèvent un taux de $82 \%$ de couverture adéquate avec une perte de vue (abandon) entre penta 1 et penta 3 égale à $14 \%$ ce qui est inférieur à l'objectif à atteindre.

L'absence d'étude spécifique sur la problématique de la couverture adéquate en penta 3 à Konobougou a motivé ce travail, qui vise à étudier les difficultés influençant la couverture adéquate en penta 3 dans l'aire couverte par le Centre de Santé Communautaire et Universitaire de Konobougou afin de proposer des alternatives pour son amélioration.

\section{MATERIEL ET METHODES}

Notre étude s'est déroulée au Centre de Santé Communautaire Universitaire de Konobougou dans le cercle de Barouéli, région de Ségou. II s'agissait d'une étude prospective type recherche action de Susman (11). L'étude s'est déroulée pendant une période de quatre mois allant du 1er avril jusqu'au 31 juillet 2018. Ont été inclus dans l'étude, l'ensemble du personnel de l'unité de vaccination, les mères/accompagnantes d'enfants à la vaccination, les relais communautaires, les leadeurs religieux, les élus locaux. N'ont pas été inclus, les personnes non consentantes.

L'échantillon comprenait 69 personnes composées de 20 usagers, représentant la population cible semestrielle du PEV rapportée à la cible nationale soit : $486 \times 4 \%$ $=19,44$ arrondis à $20 ; 10$ leaders communautaires disponibles (maire, sous-préfet, chefs de village, imam, représentante des associations féminines) ; neuf agents de santé impliqués dans la vaccination et 30 relais à raison d'un relai par village pour 20 villages et 10 relais à Konobougou ville.

Les données ont été collectées à travers une fiche d'enquête soumise aux différents participants au PEV et le renseignement d'une grille à travers l'observation (l'environnement, les tâches). Elles ont ensuite été saisies et analysées sur Epi Info version 7 et version 3.7.3.

L'étude a utilisé le modèle de recherche action de Susman qui comporte les phases suivantes : (i) Identification du problème ; (ii) Planification de l'action ; (iii) Mise en place de l'action; (iv) Evaluation des effets de l'action; (v) Partage du savoir généré.

Le consentement éclairé, après explication des objectifs du déroulement et de l'utilisation des résultats, à chaque participant de l'étude a été réquis avant l'admisinstration des outils. Ainsi, un consentement individuel écrit et signé a été obtenu de chaque sujet à l'étude. Les noms et prénoms des sujets n'étaient pas utilisés. Seul un numéro d'identification a servi d'identifier le sujet. Les données ont été gardées de façon confidentielle. Le refus d'être inclus dans l'étude n'a pas influencé la prise en charge du sujet. II s'agissait d'une étude permettant d'apporter des solutions pour résoudre les problématiques de la couverture adéquate en penta 3 , donc ne comporte aucun risque additionnel chez les sujets.

\section{RESULTATS}

Durant la période d'étude, nous avons questionné et/ou observé 69 personnes dans l'évaluation initiale et finale dont 9 agents de santé, 30 relais communautaires, 20 mères d'enfants et 10 leaders communautaires (tableau l).

\section{Renseignements généraux}

La disponibilité était de $100 \%$ conformément aux normes requises pour le personnel impliqué dans le PEV, les doses de Penta, le réfrigérateur, le porte vaccin, les accumulateurs et les consommables. Le réfrigérateur, les portes vaccins et accumulateurs étaient en bon état. II existait deux motos également en bon état pour la stratégie avancée.

Le sexe masculin était le plus représenté dans notre étude avec $60,87 \%$ dans les deux évaluations concernant les personnes enquêtées. Les personnes enquêtées résidaient dans la ville de Konobougou dans $56,52 \%$ dans les deux évaluations.

La tranche d'âge des mères d'enfants 21-30 ans représentait $65 \%$ et la tranche d'âge $41-50$ ans des relais était dominant à $53,3 \%$.

Dans notre étude, $50 \%$ des mères d'enfants étaient non scolarisées et $80 \%$ des relais communautaires avaient le niveau primaire.

Les matrones représentaient $44,4 \%$ du personnel de santé impliqué dans le PEV (figure I).

\section{Renseignements spécifiques}

Les mères d'enfants qui connaissaient la tranche d'âge du PEV représentaient $20 \%$ à l'évaluation initiale contre $75 \%$ à l'évaluation finale après le plan d'action (tableau II).

Pour les relais communautaires, cette connaissance a évolué de $66,7 \%$ à $100 \%$.

Les mères d'enfants qui n'avaient aucune idée sur les maladies cibles du penta 3 représentaient $65 \%$ au début de l'enquête contre $5 \%$ après l'intervention. 
Les mères d'enfants qui connaissaient la moitié des maladies cibles du PEV ont évolué de $0 \%$ à $55 \%$ de l'évaluation initiale à l'évaluation finale, après le plan d'action.

Nous avons noté une évolution de $20 \%$ à $80 \%$ des mères d'enfants qui ne connaissaient pas ce qu'il faut faire devant les manifestations adverses post immunisation (MAPI) entre les deux évaluations.

La connaissance des relais communautaires sur le calendrier vaccinal a évolué de $66,7 \%$ à $100 \%$ (figure II). Aucun relai communautaire ne connaissait la totalité des maladies cibles du PEV à l'évaluation initiale, soit 100\% contre $10 \%$ après l'intervention.

Nous avons constaté que $66,7 \%$ des agents de santé n'avaient pas reçu de formation en information, éducation et communication (IEC) et sur la gestion de MAPI au début de notre étude et $0 \%$ à la fin (figure III).

Le niveau d'implication des décideurs était satisfaisant dans $80 \%$, au cours des deux évaluations.

\section{DISCUSSION}

\section{Renseignements généraux}

Le sexe masculin était le plus représenté dans notre étude avec près de deux tiers dans les deux évaluations concernant les personnes enquêtées. Nous pensions que les différentes disponibilités constitueraient un bon indicateur pour l'atteinte des objectifs concernant la couverture de penta 3. Alors que, selon Boa A, la mauvaise disponibilité de ressource humaine, la rupture des vaccins et des consommables ont été énumérés parmi les principaux problèmes qui justifient l'évolution médiocre du PEV (12).

Chez les mères d'enfants, la tranche d'âge 21-30 ans représentait environ deux tiers et chez les relais celle de 41-50 ans dominait avec plus de la moitié. Cette tranche d'âge des mères nous amènerait à dire qu'elle est très active sur le plan de la procréation. Alors que la tranche d'âge des relais prouverait que ces relais commencent à vieillir.

Dans notre étude, la moitié des mères d'enfants étaient non scolarisées et quatre cinquième des relais communautaires avaient le niveau primaire. La scolarisation d'une personne serait importante pour la compréhension d'une chose. Notre résultat était légèrement inférieur à celui de Seck I et al. qui trouvent $62,5 \%$ pour les mères ou gardiennes d'enfants alphabétisées (13).

Tandis que Sackou K.J et al. pensent que comparativement aux mères jamais scolarisées, les enfants des mères de niveau supérieur et de niveau primaire ou secondaire avaient respectivement quatre et deux fois plus de chance d'être complètement vaccinés (14).

\section{Renseignements spécifiques}

Les mères d'enfants qui connaissaient la tranche d'âge du PEV représentaient $20 \%$ à l'évaluation initiale contre $75 \%$ à l'évaluation finale après le plan d'action. Pour les relais communautaires, cette compatissance a évolué de $66,7 \%$ à 100\%. Les mères d'enfants qui connaissaient la moitié des maladies cibles du PEV a évolué de $0 \%$ à $55 \%$, de l'évaluation initiale à la finale. Les mères d'enfants qui n'avaient aucune idée sur les maladies cibles du penta 3 a considérablement diminué après l'intervention. Aucun relai communautaire ne connaissait la totalité des maladies cibles du PEV à l'évaluation initiale, contre un dixième après l'intervention. La connaissance des maladies cibles du PEV, par les parents, les amèneraient à lui accorder une importance. Selon Boa A, les populations connaissaient les maladies cibles du PEV mais ne savaient pas qu'elles font l'objet d'un programme spécial appelé PEV (12). Seck I et al. obtient pour les mères ou gardiennes d'enfants $63,3 \%$ qui connaissaient les maladies cibles du PEV (13), un résultat supérieur au nôtre.

Nous avons noté une évolution de $60 \%$ de mères d'enfants qui ne connaissaient pas ce qu'il faut faire devant les MAPI, entre les deux évaluations. Nous avions pensé que la méconnaissance de la conduite à tenir devant les MAPI serait une raison d'abandon de la vaccination des enfants. Par contre, en cas de MAPI, seulement $29,8 \%$ se rendaient dans les structures sanitaires et $59,7 \%$ faisaient des soins à domicile dans l'étude de Seck I (13).

La connaissance des relais communautaires sur le calendrier vaccinal a évolué de deux tiers à la totalité. Tandis que Ndiaye NM et al. trouvent pour la connaissance du calendrier vaccinal global $38,1 \%$ des cas (2). Nous pensions que la connaissance du calendrier par les relais facilitera la recherche active des perdus de vue. Ainsi Nounawon $E$ et al. disent qu'il est important d'identifier les enfants en retard sur leur calendrier et de les considérer comme un groupe prioritaire pour une intervention de suivi actif (15)

Nous avons constaté que $66,7 \%$ des agents de santé n'avaient pas reçu de formation en IEC et sur la gestion de MAPI au début de notre étude et $0 \%$ à la fin. En révanche, Boa A pense que l'insuffisance de " l'information, de l'éducation et de la communication " (IEC) était un problème essentiel pour l'évolution normale du PEV (12).

Le niveau d'implication des décideurs était satisfaisant au cours des deux évaluations. Nous pensons que les leaders communautaires jouent un rôle primordial dans le programme de vaccination à travers la sensibilisation de la communauté, en cas de refus des parents de faire vacciner les enfants, et surtout dans la mobilisation de la population pour les séances de vaccination. Notre résultat était supérieur à celui de Yao GHA et al., dans leur étude, les leaders qui avouent n'avoir jamais participé aux activités de vaccination représentent 58,6\% (16).

\section{Points forts}

- La participation active de tout le personnel impliqué dans la vaccination, les relais communautaires et l'ASACO dans l'exécution du plan d'action ; -La disponibilité des relais communautaires, des leadeurs communautaires et le Directeur technique du centre ; 
-La maitrise de l'échantillonnage.

Points à améliorer

- La perception et le comportement des parents d'enfants ;

- La motivation des relais dans la recherche active des perdus de vue.

\section{CONCLUSION}

Cette étude a permis de poser des actions et impliquer les populations concernées de manière participative à tous les processus méthodologiques de la recherche. La 1 ère phase était consacrée à faire un diagnostic de la situation. Nous avons constaté des points forts et des points à améliorer à tous les niveaux en matière de vaccination chez les enfants de $0-11$ mois. Ainsi, des plans d'actions ont été planifiés et exécutés. II y a eu quelques changements de comportement auprès des populations concernées en matière de vaccination chez les enfants de 0-11 mois. II sera donc nécessaire : - pour l'association de santé communautaire de Konobougou : de renforcer la formation, d'encourager les relais communautaires et de surveiller l'application du plan d'action ; - pour le personnel chargé du PEV : de continuer et optimiser les séances d'IEC pratiquées dans les centres, afin d'améliorer les connaissances des populations en matière de vaccination.

\section{Références}

1. N M Ndiaye, $\mathbf{P}$ Ndiaye, A Diédhiou, A S Guèye, A T Dia : Facteurs d'abandon de la vaccination des enfants âgés de 10 a'23 mois à Ndoulo (Sénégal). Cahiers Santé 2009 ; vol. 19, n $1: 9-13$.

2. I Pruvosta, S. Lowingerova-Fauveta, P. Debackera, B. Dervauxa, F. Dubosa, A. Martinota : Evaluation de l'application du calendrier vaccinal de l'enfant en médecine libérale. Archives de Pédiatrie 2012 ;19 : 248-253.

3. M Cayemittes, A Chahnazarian : Survie et santé de l'enfant en Haïti. Institut Haitian de L'Enfance, Port-au-Prince. Éditions de l'enfance 1989 ; 82-95.

4. M Kacem, W Dhouib, C Bennasrallah, I Zemni, H Abroug, M Fredj, A S Belguith : Programme élargie de vaccination aux pays du maghreb. etude de cas de la tunisie. Revue systématique de la littérature. LA TUNISIE MEDICALE - 2018 ; 96 : 696-705.

5. M.-A. Balinskaa, C. Léona : Opinions et réticences face à la vaccination. La Revue de médecine interne $2007 ; 28$ : 28-32.

6. F M Randriatsarafara, S. Ralamboson, El-C J Rakotonirina, H Rahoelison, R J Ranjalahy, R H
Ratsimbaz : Respect du calendrier vaccinal selon le programme élargi de vaccination au CSMIU de Moramanga. Rev. méd. Madag. 2014 ; 4(2) : 457-463.

7. Section immunisation/Direction nationale de la santé : Grille de la supervision nationale de la vaccination de routine dans les régions de Koulikoro, Ségou et Mopti 2015.

8. M Dionne, N Boulianne, B Duval, F Lavoie, N Laflamme, J Carsley, L Valiquette, S Gagnon, L Rochette, G D Serres: Manque de conviction face à la vaccination chez certains vaccinateurs québécois. Revue Canadienne de Santé Publique 2001 ; 92, NO. 2 : 100104

9. P. Talani,J. Nkounkou-Pika,H. Mayanda, F. Yala : Les occasions de vaccination manquées à Brazzaville. Bull Soc Pathol Exot 2000 ; 93, 2 : 121-122.

10. Enquête Démographique et de Santé du Mali (EDSM-V)2012-2013. 547 p.

11. Gabriel G, Lessard-Hébert M : La recherche action : Ses fonctions, son fondement et son instrumentalisation. Presse de l'Université de Québec. 1987 ; 125p.

12. A. Boa : Perceptions du Programme élargi de vaccinations (PEV) et de ses dysfonctionnements dans le district sanitaire de Bouna (Nord-Est de la Côte d'Ivoire). Bull Soc Pathol Exot, 2006 ; 99, 5 : 386-390.

13. I Seck, B Diop, M M Leye, B M Mboup, A N'diaye, P A Seck et al : Déterminants sociaux de la couverture vaccinale de routine des enfants de 12 à 23 mois dans la région de Kaolack, Sénégal. Santé Publique $2016 ; 28: 807-815$.

14. K.J. Sackou - A.S.S. Oga - A.A. Desquith · Y. Houénou - K.L. Kouadio : Couverture vaccinale complète des enfants de 12 à 59 mois et raisons de nonvaccination en milieu périurbain abidjanais en 2010. Bull. Soc. Pathol. Exot. 2012 ; 105 : 284-290.

15. E Nounawon, G D Serres, N Boulianne, B Duval : Impact d'une recherche active d'information vaccinale chez les enfants ayant un carnet de vaccination incomplet ou chez ceux qui l'ont perdu. Canadian journal of public Heath $2001 ; 267-271$.

16. G H A Yao, L B N Aka, N J M Manouan, K 0 Angbo-Effi, A Douba, P Zengbé-Acray : Connaissances et attitudes des organisations de la société civile à la mise en œuvre du Programme élargi de vaccination de routine en Côte d'Ivoire. Santé Publique 2014 ; 26 : 99-106. 
Liste des tableaux et figures :

Tableau I : Relatif aux personnes enquêtées pendant notre étude

\begin{tabular}{lcc}
\hline Qualification des personnes enquêtées & Effectif & $\%$ \\
\hline Agent de santé & 9 & 13,0 \\
Relais communautaires & 30 & 43,5 \\
Mère & 20 & 29,0 \\
Leaders religieux & 10 & 14,5 \\
Total & 69 & 100,0 \\
\hline
\end{tabular}

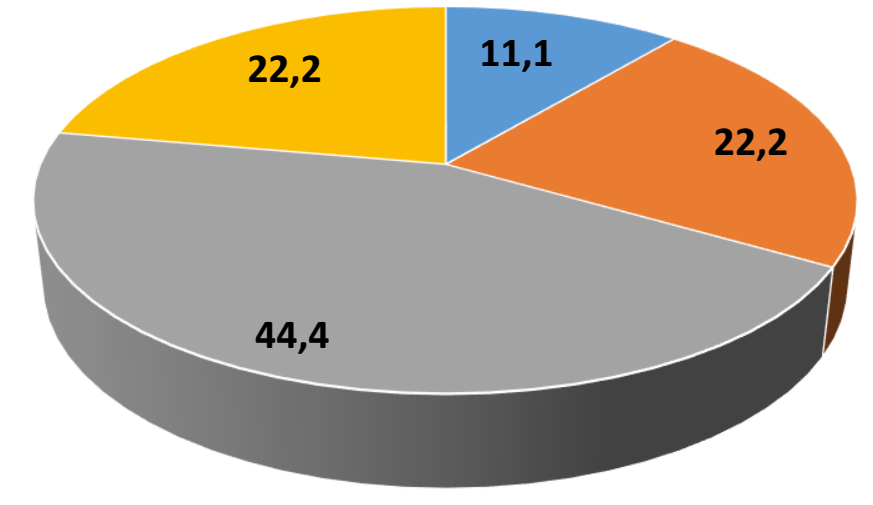

$$
\square \text { Aide-soignant } \quad \text { Infirmier (e) } \quad \text { Matrone } \backsim \text { Sage-femme }
$$

Figure 1 : Répartition des agents de santé selon leur qualification

Les matrones représentaient 44,4\% du personnel impliqué au PEV

Tableau II : Répartition des mères d'enfants selon leur niveau de connaissance sur la tranche d'âge du PEV

\begin{tabular}{lcc|cc}
\hline & Avant & & \multicolumn{2}{c}{ Après } \\
\hline & Effectif & $\%$ & Effectif & $\%$ \\
Oui & 4 & 20,0 & 15 & 75,0 \\
Non & 16 & 80,0 & 5 & 25,0 \\
Total & 20 & 100,0 & $\mathbf{2 0}$ & 100,0 \\
\hline
\end{tabular}

Les mères d'enfants qui connaissaient la tranche d'âge du PEV représentaient $20 \%$ à l'évaluation initiale contre $75 \%$ à l'évaluation finale.
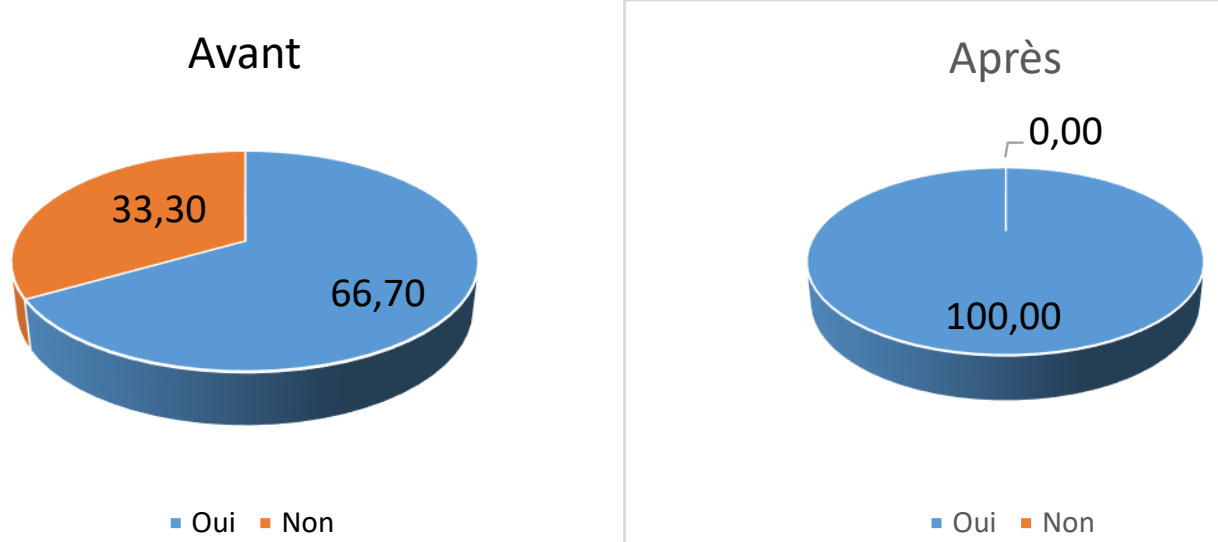

Figure 2 : Répartition des relais communautaires selon leur niveau de connaissance sur le calendrier vaccinal Les relais communautaires qui connaissaient le calendrier vaccinal représentaient $66,7 \%$ à l'évaluation initiale contre $100 \%$ à l'évaluation finale. 


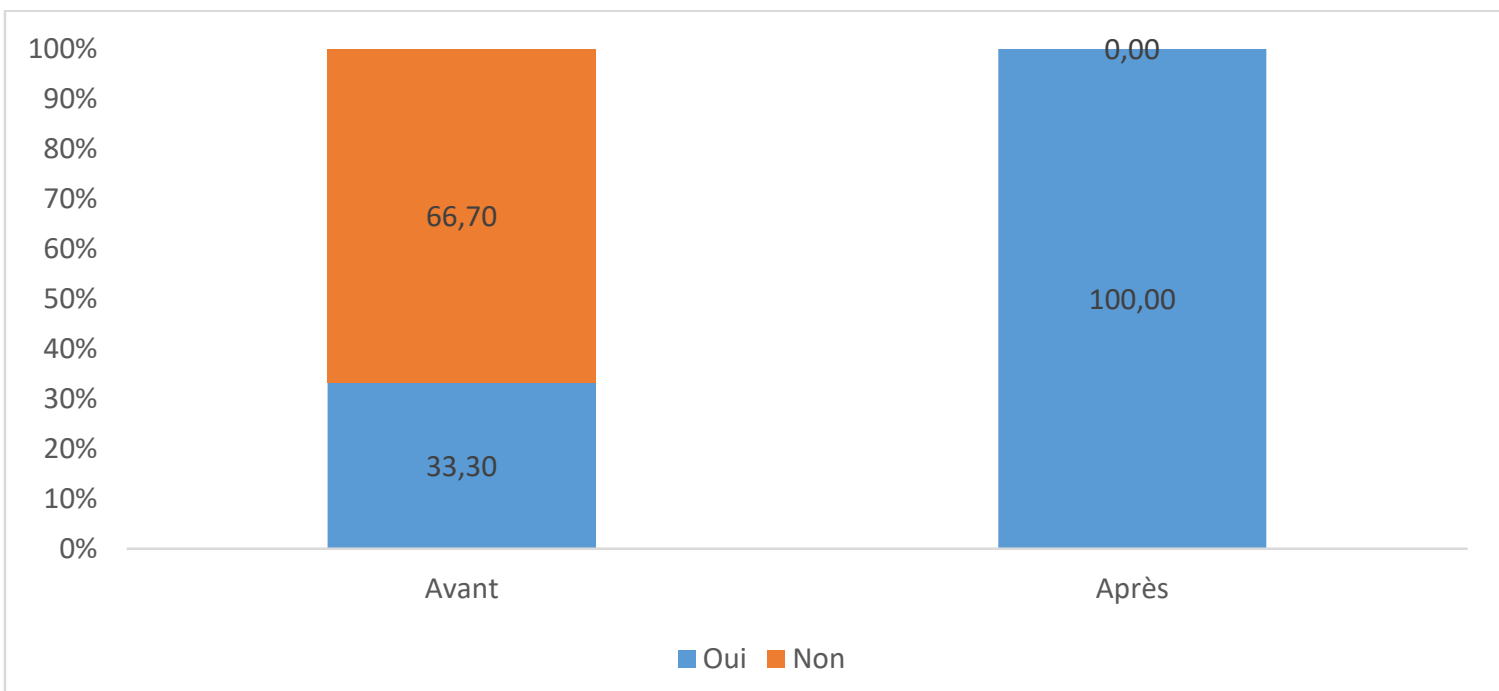

Figure 3 : répartition des agents de santé en fonction de la formation reçue en IEC

Les agents de santé qui ont reçues la formation en IEC a évolué de 33,3 à $100 \%$ après le plan d'action. 